\title{
Innovative Configuration for a Far Infrared Space Interferometer
}

\author{
V.A.Iafolla ${ }^{1,2}$, E.Fiorenza ${ }^{1,2}$, L.Iafolla ${ }^{1}$, C.Lefevre ${ }^{1,2}$, C.Magnafico ${ }^{2}$, F.Santoli ${ }^{1,2}{ }^{\text {, G.Savini }}{ }^{3}$ \\ 1 AGI - Assist in Gravitation and Instrumentation srl, Rome, Italy \\ Valerio.Iafolla@agi-tech.com
}

2 IAPS - Institute for Space Astrophysics and Planetary Sciences, INAF, National Institute of Astrophysics, Rome, Italy

3 University College London, London, UK

http://www.homepages.ucl.ac.uk/ucapgsa.html

\begin{abstract}
In the last ten years many proposal and study were been advanced for a far-IR kilometer baseline interferometer. This paper shows some results concerning FISICA (Far Infrared Space Interferometer Critical Assessment) an FP7 program of the European Community. In particular, we focus on an innovative strategy of observation to cover the $u, v$ plane of observation with a minimal consumption. Will be showed the numerical simulation results for a three booms configuration.
\end{abstract}

Keywords - FISICA, FP7, interferometer, strategy, far-IR.

\section{INTRODUCTION}

In the last decade many studies have been performed for a Far Infrared space telescope. This kind of instruments allows to observe wavelength radiations from some of colds and most distant objects in the Universe. Earth's atmospheric absorbs a lot of part of IR radiation, so to see the IR band is mandatary build infrared telescope in the space. Thus would be a limit to the angular resolution, indeed the angular resolution it directly proportional to the diameter of the mirror telescope. To bypass the limit imposed by the space vector housing (e.g. James Webb Space Telescope will be $6.5 \mathrm{~m}$ of diameter), it is used the synthetic aperture. Thanks two this technique, two little mobile telescope (e.g. $2 \mathrm{~m}$ of diameter) have the same angular resolution of a big one fixed (e.g. $100 \mathrm{~m}$ of diameter) on the condition that they recovered the same space which the big one occupies, during their evolutions.

In the wake of other mission hypothesis like SPECS [1], SPIRIT [2], FIRI [3], in 2013 the European Community has financed a three years' development program called FISICA [4] (Far Infrared Space Interferometer Critical Assessment). It pursues to define the instrument requirements for a double Fourier modulation payload while advancing the beam divider technology for a range of operation of 30-300um.

One of the principal issues for any study is the tradeoff between large baseline, dynamic control and low consumption.
This article proposes a hypothetical configuration to guarantee large synthetic aperture $(100 \mathrm{~m})$ with a quite zero consumption. It will be show as this innovative configuration realizes a constant moment of momentum dynamic while the two satellites tread all the plain of view changing their baseline.

\section{CONFIGURATION}

For a Michelson interferometer the angular resolution is determined by the separation, or "baseline," between the light collecting mirrors. Each baseline provides information about the source on a particular spatial frequency. The longer the baseline, the higher the frequency. For this simulation has been choice a maximum baseline of $100 \mathrm{~m}$. For our simulation we have choose a multibody calculation program in a concentered parameters model. This kind of model doesn't allow deformation of the structure but it is useful to see the dynamic and the free evolution of the system.

Moreover, FISICA as the other interferometric telescopes, are designed to be positioned in Lagrangian point L2 in Sun-Earth reference frame. As known in that point the object remain fixed respect to the Sun-Earth reference and the gravity and gravity gradient are quite zero. For this reason, the gravity is not considered in our simulation.

This configuration forecasts three booms and three satellites. As in SPIRIT configuration the first satellite, called Collider (Hub0), is in the center of the scheme, where the two beams will be combined. This Collider is joined to a long boom (B1) of $50 \mathrm{~m}$ long. In the SPIRIT concept the other two satellites change their baseline using this boom like a rail. In our idea the other two satellite (Hub1 and Hub2) are not directly linked to the principal boom (B1). There are two other booms (B2 and B3) linked at the tips of B1 by means two hinges. B2 and B3 are long almost half then boom1 $(23 \mathrm{~m})$. Hub1 and Hub2 are joined to the edge of B2 and B3 as shown in figure (1). The Collider satellite has a mass of $325 \mathrm{~kg}$ and Hubs of $1000 \mathrm{~kg}$ each. One of the most important aspects for this kind of 
configuration is the orientation of principal moment of inertial. Indeed, to be secure that the rotation axes remain parallel to the initial pointing axes, all the principal moment of inertia have to start with the same attitude. Otherwise will be not possible obtain a stable motion but it degenerates in a chaotic one. The same importance has the perfectly centering of the center of mass. It has to be positioned at the center of the geometrical scheme. Any misalignment respect to center of mass or principal moment of inertia have to be compensate with a specific procedure of control, moving auxiliary masses, that is out of scope of this paper.

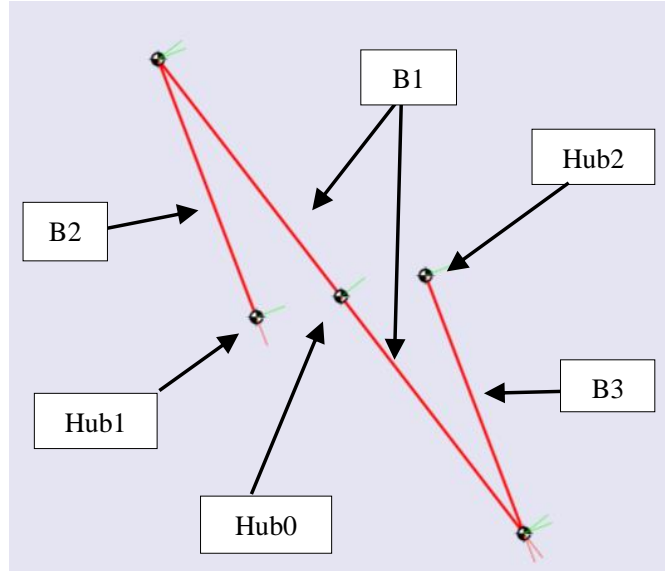

Figure 1 Scheme of Interferometer configuration.

At the starting point the two Hubs and the Collider are aliened and the two booms are closed (see fig.2).

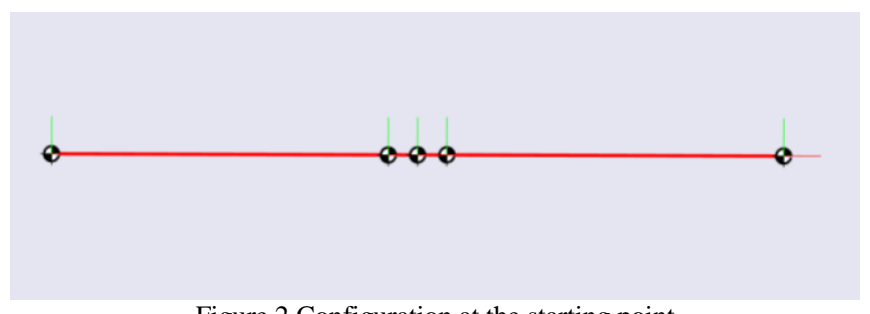

Figure 2 Configuration at the starting point

To initialize the free evolution a starting energy has to be provided. With respect to the values of masses and inertia used for this simulation we have provided a $10 \mathrm{~N}$ for 40 s by means two thruster fixed to Hub1 and Hub2 and ever oriented orthogonally to $\mathrm{B} 1$ and $\mathrm{B} 2$ respectively, with opposite verse (see fig.3).

During the starting period the two Hubs began to increase our velocity until to have an absolute velocity of $0.04 \mathrm{~m} / \mathrm{s} * \mathrm{~s}$. During this period even the B2 an B3 increase their angular velocity. For the action reaction principle also B1 increase its absolute angular velocity but with opposite sign (see fig.4). After the first 40s the starter is switched off. At that time, we have reached the initial conditions for system. B2 and B3 will have the same angular velocity indeed B1 has a different one.
We can change the ratio between the angular velocity of B1 respect $\mathrm{B} 2$ or $\mathrm{B} 3$ changing the ratio of inertial moment.

\section{FREE EVOLUTION}

After the thruster switch off the system began its free evolution. Due to the symmetry of the configuration geometry, the center of mass of all the system does not change position. It remains fixed in the center of the geometer.

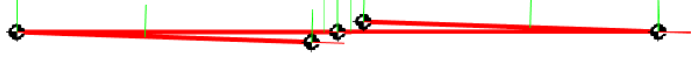

Figure 3 Configuration at the switch off

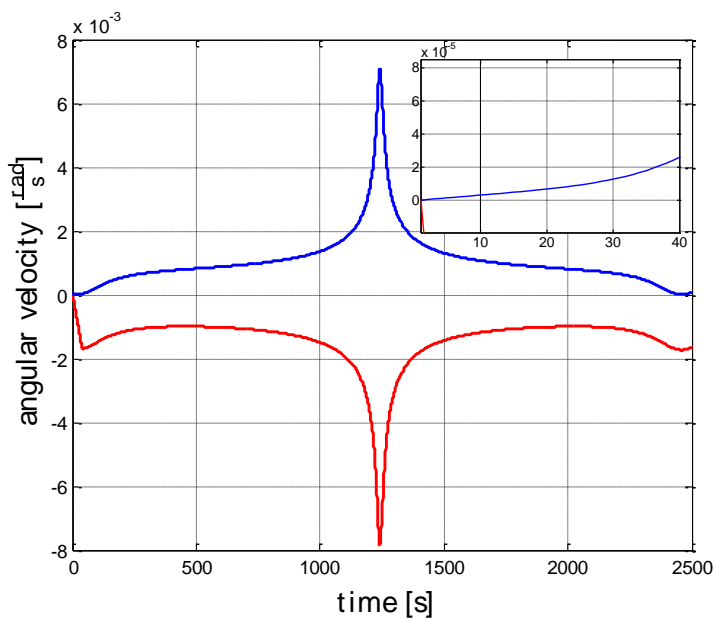

Figure 4 Periodicity of angular velocity of B1 (blue line) and B2 B3 (red line). Particular of B1 angular velocity during the switch on period (up to right).

The free evolution (see fig.5) shows how the paths of two satellites cover quite completely the plane of measuring. Consideration on the cover efficient respect to time can be done. Changing the ratio of mass between Hab0 and Hub1, Hub2 it is possible to change the time of recovering but any further is linked with future constructive aspect. In any case the consumption quite zero for all the time of the free evolution. In this scheme the only consumption which has to estimate is for the control of non-perfectly balancing and repointing of the apparatus. 

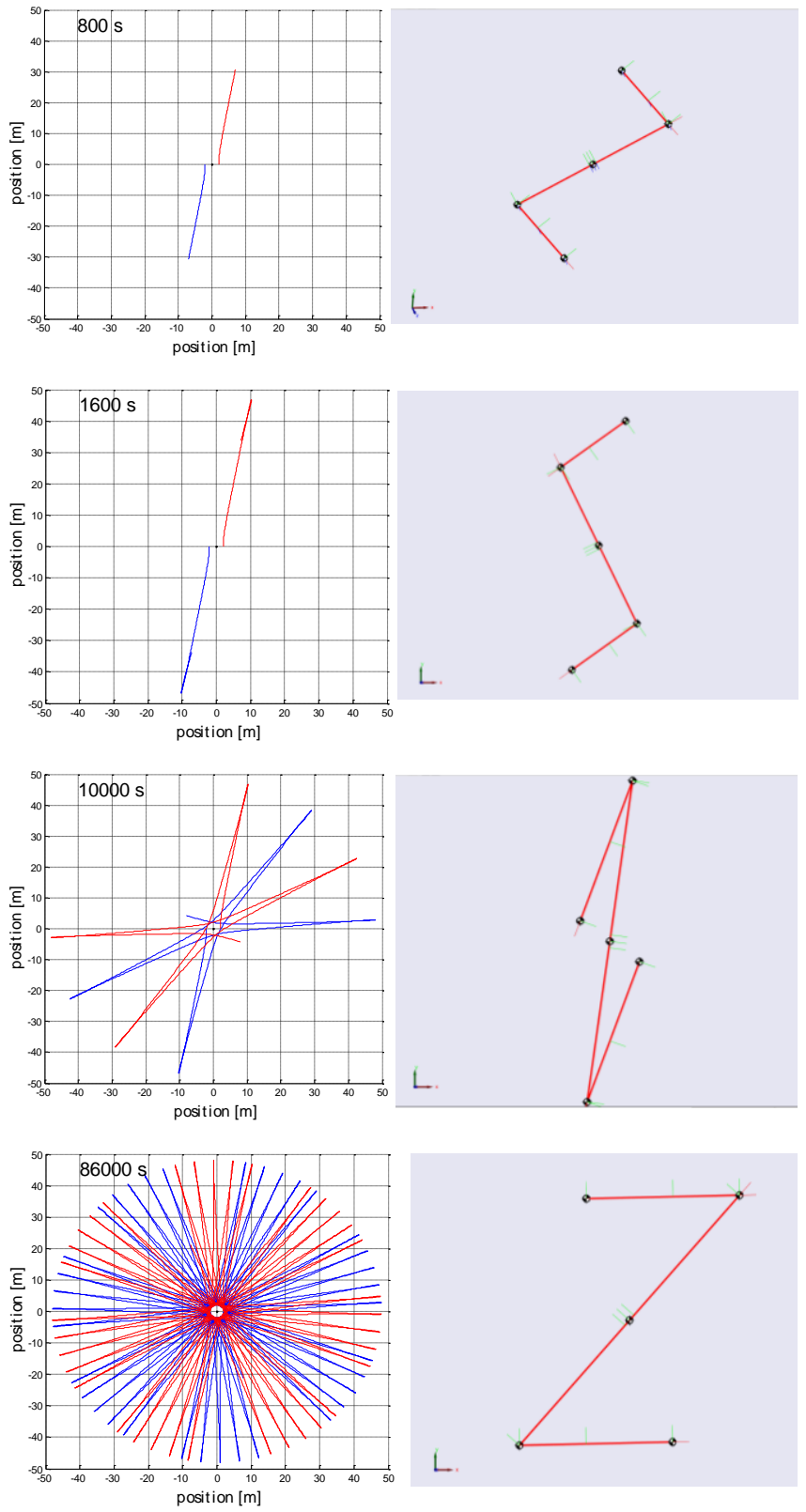

Figure 5 Time sequence for 800s 1600s 10000s 86000s. Path of Hub1 (blue) and Hub2 (red) on left. Actual system configuration, on right.

One aim of this geometry is that the two satellites have a quite constant absolute velocity during the plane covering. It is necessary to have the same time of exposition for each baseline. Due to the starting condition the Hubs will have an average velocity of $0.04 \mathrm{~m} / \mathrm{s}^{*} \mathrm{~s}$ for all the time. It means that every 25 seconds the Hubs telescopes run through $1 \mathrm{~m}$ which is the Hub's mirror diameter. Thus time is necessary to gathering a sufficient amount of photons for each acquisition point. As shows the Fig. 6 the velocity vs baseline is quite constant for baseline range $2 \mathrm{~m}$ to $70 \mathrm{~m}$. After $70 \mathrm{~m}$ the velocity decrees. This kind of beaver guarantees to have more acquisition time for the high frequency signals.

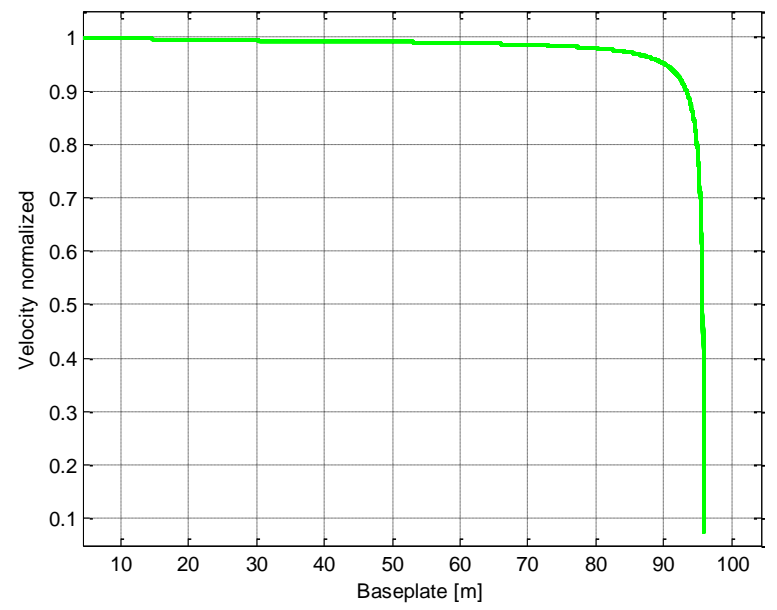

Figure 6 Absolute velocity (normalized with $0.04 \mathrm{~m} / \mathrm{s} * \mathrm{~s}$ ) of Hab1 versus distance between Hub1 and Hub2 (baseline)

\section{CONCLUSIONS}

Has been presented a new FIR space interferometer permitting an observation strategy that offer the opportunity to Respect to other infrared interferometer telescope scheme, the two satellite must not be actuated to change their baseline. It has a obvius impact on the consuption, but is impotance on the noise of the apparatus too. Indeed any active froce used to chenge the ralative distance between the Hubs will introduce displacement perturbtions and metrological problems. In ouar scheme all the dinamic is passive and non external noise is injected.

Andrebbe indicato qualche problema connesso a questa nuova modalità, esempio: come la luce viene convogliata all'HUB centrale con l'attraversamento dei pivot.

\section{REFERENCES}

[1] Leisawitz D.T., Danchi W.C., DiPirro M.J., Feinberg L.D., Gezari D.Y., Hagopian M., Langer W.D., Mather J.C., Moseley S.H., Shao M., Silverberg R.F., Staguhn J., Swain M.R., Yorke H.W., Zhang X., "Scientific motivation and technology requirements for the SPIRIT and SPECS far-infrared/submillimeter space interferometers", in: Breckinridge, J.B., Jakobsen, P. (Eds.), UV, Optical, and IR Space Telescopes and Instruments. Proc. SPIE, vol. 4013. SPIE - The International Society for Optical Engineering, Bellingham, WA, pp. $36-46,2000$

[2] Wilson M.E. et al. "The Space Infrared Interferometric Telescope (SPIRIT): Optical System Design Considerations" (2007).

[3] ESA report, "FIRI, Far Infrared Interferometer," ESA CDF Study Report CDF-49(A) (2006).

[4] G.Savini, P.A.R. Ade, N.Baccichet, C.Bracken, K.Dohlen, A.Donohoe, B.Gom, M.J. Griffin, W.Holland, V.Iafolla, R.J. Ivison, M.Jones, R.Juanola-Parramon, J.Lightfoot，S.Liu, A.McMillan , J.A.Murphy, 
D.Naylor, C.O'Sullivan , E.Pascale , S.Pezzutto , E.Rakotonimbahy , D.Schito , L.Spencer , L.Spinoglio , B.Swinyard , I.Venendaal , S.Vives , D.Walker, And D.Leisawitz , S.Shi , H.Matsuo, "Progress in the Critical Assessment for a Far-Infrared Space Interferometer with Double Fourier Modulation (FP7-FISICA)' 